\title{
Der jetzige Bleigehalt der Vegetation in der Nähe schweizerischer Autostraßen
}

Th. Keller

Artikel eingegangen am 16. Februar 1970

August 1970, Nr. 48
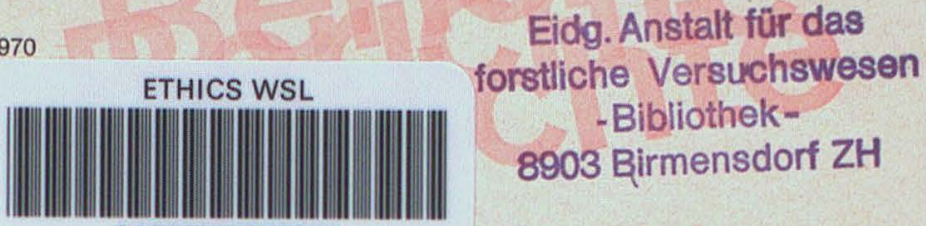

01000000595376

\section{Zusammentassung}

Die vorliegende Arbeit gibt einen Uberblick über dit Verunreinigung der Vegetation durch die teinzerstäubten Bleiverbindungen der Auspuffgase von Benzinmotoren. Die wichtigsten Faktoren, welche den Bleigehalt der Vegetation beeinflussen, werden diskutiert, nämlich

Verkehrsfrequenz, Nadelalter und Jahreszeit, Oberflächenbeschaffenheit der pflanzlichen Organe sowie Horizontal- und Vertikaldistanz von der Straße.

Der Bleigehalt des Bodens dagegen spielt kaum eine Rolle, da nur ein Bruchteil der Bleiverbindungen im Boden von den Wurzeln aufgenommen werden kann. Oberdies ist Blei innerhalb der Pflanze nur schwer beweglich.

Der gröBte Teil der gefundenen Bleimengen haftet äuBerlich, ist jedoch nur teilweise abwaschbar.

Da die Bleikonzentration ungewaschener Agrarprodukte aus der Umgebung stark frequentierter Autostraßen nur selten unter 1 ppm sank, wird gefolgert, da $\mathrm{B}$ die Bleialkyle durch ungefährlichere Additive ersetzt werden sollten.

\section{Einleitung}

Im Frühjahr 1966 traten an Koniferen in der Ostschweiz chlorotische Nadelverfärbungen auf, welche sich längs der Straßen zu häufen schienen. Als die forstliche Versuchsanstalt beauftragt wurde, zu untersuchen, ob es sich bei diesen Erscheinungen um Auswirkungen von Motorfahrzeugabgasen handle, stellte sich uns daher die Frage, wie eine derartige verkehrsbedingte Luftverunreinigung überhaupt zu erfassen sei. Die Auspuffgase setzen sich ja aus zahlreichen, zum Teil unschädlichen Komponenten zusammen. Angesichts der überwiegenden Dominanz der Benzinmotoren im Verkehr wurde beschlossen, den Bleigehalt der Vegetation als Indikator der verkehrsbedingten Luftverunreinigung heranzuziehen. Blei ist normalerweise in den Pflanzen nur in geringen Spuren enthalten, da es für die Pflanzen kein lebensnotwendiges Nährelement darstellt.

Die dem Benzin als Klopfbremsen zugefügten Bleialkyle führen dazu, daß die Auspuffgase der Benzinmotoren bis zu $60 \mathrm{mg} / \mathrm{m}^{3}$ mehr oder weniger fein verstäubter Bleiverbindungen enthalten (Brunner, 1966). Menge und Partikelgröße hängen jedoch stark von der Fahrweise ab. Durchschnittlich ein Viertel des Bleis wird in Motor, Auspuffsystem und Ól abgelagert. Die übrigen $75 \%$ werden fortlaufend ausgepufft, wobei bei konstanter Fahrgeschwindigkeit etwa $75 \%$ eine Korngröße unter $5 \mu$ besitzen, daher verwirbelt und gasähnlich in der Atmosphäre verteilt werden, während der Rest zufolge seiner Korngröße sedimentiert (Brunner, 1966).

Als Untersuchungsobjekt wurden Fichtennadeln ausgewählt, weil nur geringe Bleimengen erwartet wurden und da vermutet wurde, die relativ große spezifische Oberfläche der mehrere Jahre ausdauernden $\mathrm{Na}$ deln würde zu gut meßbaren Konzentrationen führen. Die Bleigehaltsbestimmungen wurden von der EMPA (Dr. H. Preis) durchgeführt (für methodische Einzelheiten vgl. Keller und Preis, 1967).

Auf Grund der unerwartet hohen gefundenen Bleigehalte beauftragte die Eidgenössische Kommission für Lufthygiene die Landwirtschaftliche Forschungsanstalt Lausanne und die Agrikulturchemische Forschungsanstalt Liebefeld, 1967 und 1968 die Untersuchungen auf landwirtschaftliche Produkte auszudehnen. Herrn Direktor Dr. Bovay danke ich herzlich für die Überlassung des im Druck stehenden Manuskriptes mit den Analysenresultaten (Quinche, Zuber und Bovay, 1969).

\section{Die Entwicklung der Bleisituation}

Bekanntlich wurde 1947 der Zusatz von Bleitetraäthyl zum Autobenzin in der Schweiz bewilligt und gleichzeitig eine Kommission beauftragt, die Auswirkungen der Benzinverbleiung zu verfolgen. Die Resultate der Untersuchungen des Bleigehaltes in verschiedenen Stäuben in Zürich sind im Bericht dieser Bleibenzin-Kommission (1961) 
und im zweiten Bericht der Lufthygiene-Kommission (Högger, 1968) enthalten. Es zeigte sich, daß im Straßenstaub der Bleigehalt sehr rasch von $0,5 \%$ auf $2 \%$ anstieg und dann auf diesem Niveau blieb, während der Gesimsestaub in derselben Zeit von $0,3 \%$ auf $1,8 \%$ anstieg, wobei erwartet wird, daß er bei etwa $2 \%$ ein Gleichgewicht erreichen wird.

Koproporphyrin-Untersuchungen im Urin verschiedener Bevölkerungsgruppen blieben negativ, und der Bleigehalt im Blut blieb bei etwa $0,25 \mathrm{ppm}$ für Büroangestellte und etwa $0,35 \mathrm{ppm}$ für Garagenarbeiter (Högger, 1968). Vergleichsweise sei erwähnt, daß Patterson (1965) auf Grund geochemischer Untersuchungen und Oberlegungen zum Schluß kam, die Einwohner der USA seien von einer chronischen Bleivergiftung bedroht. Die Bleikonzentration im Blut (im Mittel 0,25 ppm; Spanne 0,05-0,4 ppm) sei bedrohlich nahe an den Grenzwert einer Bleivergiftung $(0,5-0,8 \mathrm{ppm})$ herangerückt. Er forderte daher die Beseitigung der wichtigsten Quellen der Umweltsverbleiung, unter anderem auch der Benzinverbleiung.

Aber auch in pflanzlichen Geweben nahm der Bleigehalt im Laufe der Zeit zu. Obwohl Blei in der Pflanze schwer beweglich ist (Leh, 1966; Suchodoller, 1967) fanden Schroeder und Balassa (1961) im Stammholz einer amerikanischen Alleeulme in den Jahrringen zunehmende Bleigehalte. Eine ähnliche Bleigehaltszunahme entdeckte Meier (1966) in den Jahrringen zürcherischer Fichten, welche 1954 aus einer verkehrsfernen Baumschule an eine Durchgangsstraße versetzt worden waren:

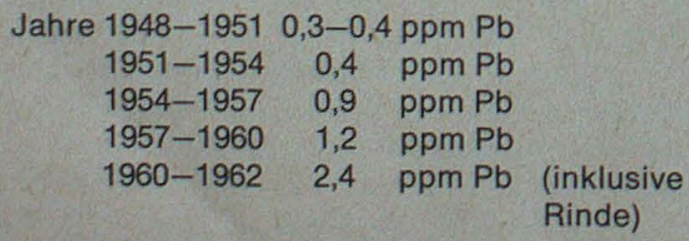

Bleizunahmen in der Vegetation Südschwedens wiesen neuestens auch Rühling und Tyler (1968) nach. Sie analysierten herbarisierte und frische Moose verkehrsferner Standorte und stellten fest, daß deren Bleigehalte von etwa $20 \mathrm{ppm}$ (um 1860) vor allem nach 1950 mit der starken Zunahme des Motorverkehrs bis 1968 auf $80-90$ ppm angestiegen waren.

Dieselben Autoren berechneten auch, daß in einem $50 \mathrm{~m}$ breiten Streifen seitlich einer Straße in Boden und Vegetation eine geringere Bleimenge gespeichert sei, als auf Grund des Verkehrs zu erwarten wäre. Sie schlossen daraus, daß der Wind einen Teil des Bleis weitertransportiere. Sie stellten einen regionalen Bleigradienten in SW-NERichtung von Süd- bis Mittelschweden fest. Das deutliche Bleigefälle von SW nach NE läßt auf eine Zuwehung von Blei aus Deutschland und Dänemark schließen. Schon früher hatten Tatsumoto und Patterson (1963) großräumige Bleiverfrachtungen nachgewiesen, da sie in den abgelegenen Bergen Kaliforniens $1,6 \mu \mathrm{g}$ Blei/kg Schnee fanden.

Aber nicht nur die erwähnte schwedische Arbeit untersuchte den Bleigehalt der Vegetation in Straßennähe. Bereits 1962 hatten Cannon und Bowles in den USA hohe Bleikonzentrationen in Gras und Gemüse entdeckt, und auch die Untersuchungen von Kloke und Riebartsch (1964), Leh (1966) sowie Suchodoller (1967) ergaben abnormal hohe Bleigehalte straßennaher Vegetation. Wohl die alarmierenden Analysenresultate aus Deutschland führten dazu, daß die Futtermittel-Kommission der EWG als duldbare Blei-Höchstmenge in der Trockensubstanz von Viehfutter $10 \mathrm{ppm}$ vorschlug (Leh, 1966). Bezüglich menschlicher Nahrungsmittel sei erwähnt, daß die Mediziner Schroeder und Balassa (1961) Analysen kanadischer und englischer Pflanzen aus der Nähe von Autostraßen zitieren (Warren und Delavault), welche bei Gerste 1-6 ppm und bei Kohl 
1-3 ppm Blei ergaben, Werte, die sie als oberhalb der Sicherheitsgrenze für Lebensmittel liegend erachteten. Die Eidgenössische Lebensmittelverordnung nennt keine Bleigehaltsgrenze für Nahrungsmittel, so da $B$ offenbar höchstens Spuren dieses Metalls in Lebensmitteln enthalten sein dürfen! Für Schönungs-, Konservierungsmittel und dergleichen, welche nur in kleinen Mengen den Lebensmitteln beigegeben werden, nennt diese Verordnung in der Regel $10 \mathrm{ppm}$ Blei als obere Grenze, so daß sie zu höchstens einem Bruchteil eines ppm Blei im behandelten Nahrungsmittel führen.

\section{Faktoren, welche den Bleigehalt beeinflus-} sen

\subsection{Verkehrsfrequenz}

Schon den wenigen Analysendaten von Kloke und Riebartsch (1964) ist ein Einfluß der Verkehrsdichte auf den Bleigehalt der Vegetation zu entnehmen. Da unsere Untersuchung Fichten an Straßen verschiedenster Ver-

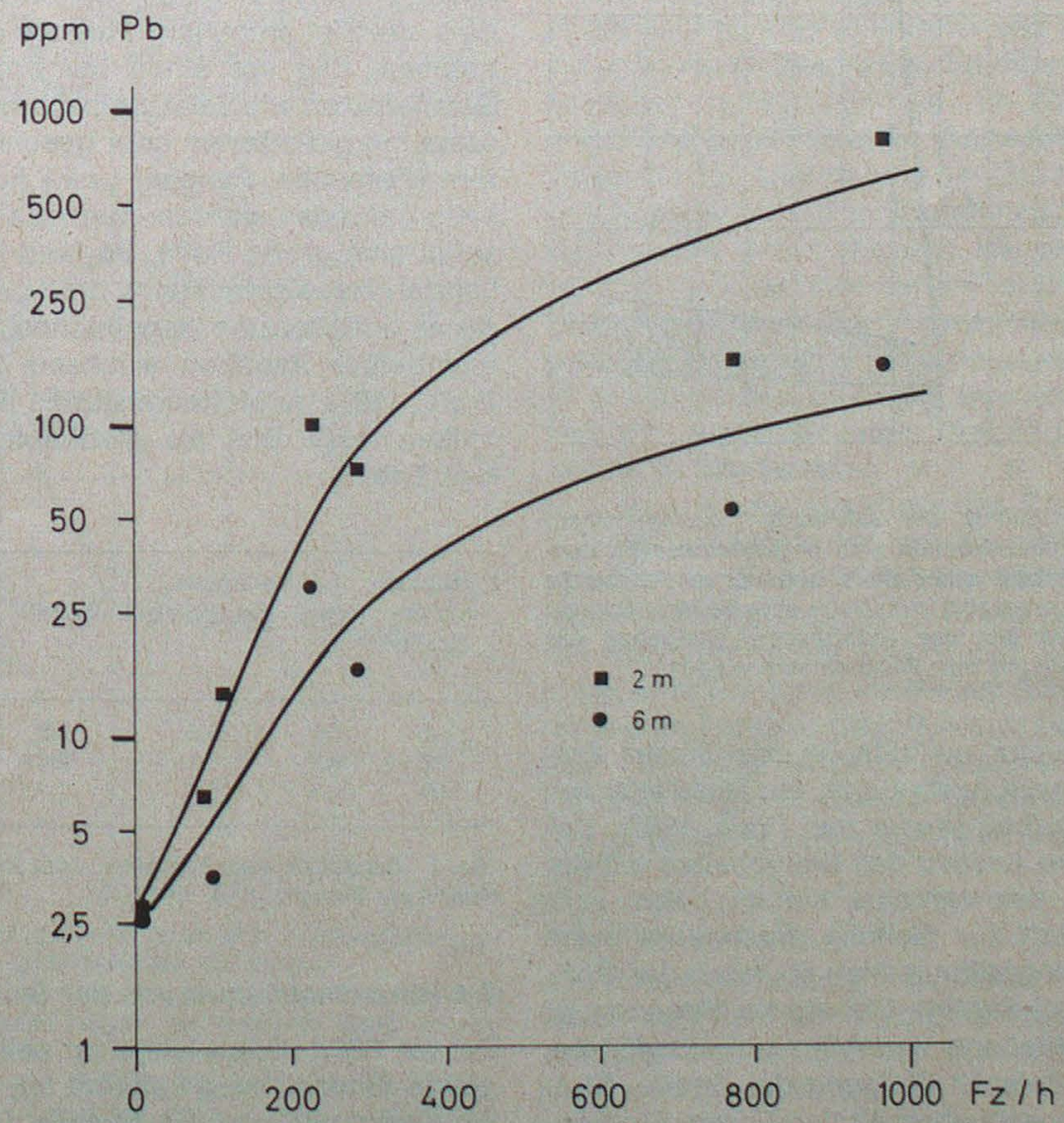

Abb. 1 Der Einfluß der Verkehrsdichte (Fahrzeuge/h) auf den Bleigehalt in der Trockensubstanz ungewaschener, ein- und zweijähriger Fichtennadeln in $2 \mathrm{~m}$ und $6 \mathrm{~m}$ Höhe über Boden (aus Keller und Preis, 1967). 


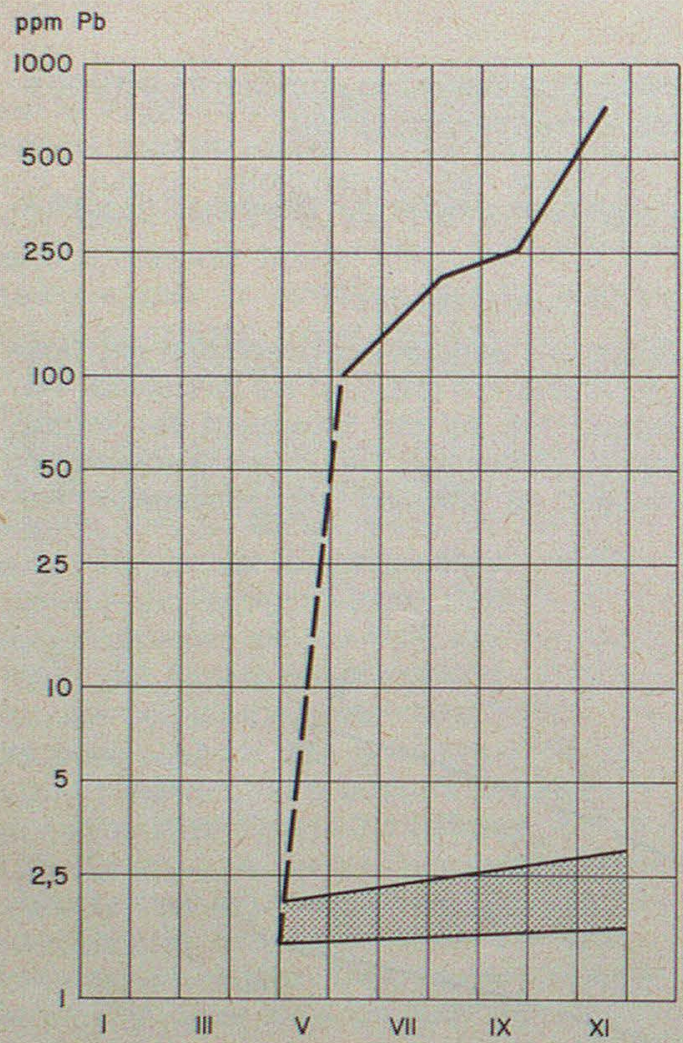

Abb. 2 Der EinfluB der Jahreszeit (Expositionszeit) auf die Bleikonzentration sich entwickelnder Fichtennadeln am Rand einer stark befahrenen Autostraße (rund 770 Fchrzeuge/h; $1 \mathrm{~m}$ Höhe über Boden). Das gerasterte Band gibt den snatürlichen" Bleigehalt von Fichtennadeln aus dem Waldesinnern wieder.

kehrsfrequenz umfaßte, wurde dieser Einfluß überprüft, wobei sich die Resultate von Abb. 1 ergaben (Keller und Preis, 1967). Der sehr starke Anstieg des Bleigehaltes mit der Zunahme des Verkehrs kommt dabei sehr eindrücklich zur Geltung, besonders wenn man den logarithmischen Maßstab der Ordinate berücksichtigt. Die starke Streuung ist auf verschiedene Variablen zurückzuführen, wie zum Beispiel Steigung der Straße, StraBenbreite, seitlicher Abstand vom Straßenrand, Bewindung usw.
3.2 Nadelalter (Expositionsdauer) und Jahreszeit

An jungen Fichtennadeln ließ sich von Mai bis November sehr schön ein kontinuierliches Ansteigen des Bleigehaltes verfolgen (Abb.2), während sich an alten Nadeln keine ausgeprägten Unterschiede mehr zeigten.

\subsection{Oberflächenbeschaffenheit der Pflanzen}

Je nach der Beschaffenheit der Blattoberfläche wird mehr oder weniger Blei angelagert. So zeigten die Untersuchungen von Rühling und Tyler (1968), daß Moose besonders viel Blei enthalten. Quinche et al. (1969) betonen, da $\beta$ vor allem bei breitblättrigen Gemüsearten wie Salat, Spinat usw. sowie bei Arten mit gefiederten oder geschlitzten Blättern (Petersilie, Fenchel usw.) hohe Bleigehalte gefunden werden. Auch die Behaarung spielt eine große Rolle. So fand sich an behaarten Haselnußblättern dreimal mehr Blei als an unbehaarten Hagebuchenblättern, die unmittelbar daneben wuchsen (Keller und Preis, 1967). Auch flaumhäutige Früchte enthielten mehr Blei als glatthäutige Früchte (vgl. Tab. 1).

\begin{tabular}{ccccc}
\hline $\begin{array}{c}\text { Entfernung } \\
\text { von der } \\
\begin{array}{c}\text { Straße } \\
m\end{array}\end{array}$ & $\begin{array}{c}\text { Aprikosen } \\
\text { Haut }\end{array}$ & Fruchtfleisch & Schale & $\begin{array}{c}\text { Birnen } \\
\text { Fruchtfleisch }\end{array}$ \\
\hline 5 & 7,5 & 1,1 & 3,9 & 0,7 \\
15 & 6,9 & 1,1 & 2,0 & 0,6 \\
50 & 5,8 & 1,1 & 1,1 & 0,4 \\
\hline
\end{tabular}

Tab. 1 Bleigehalt von Früchten, $\mathrm{ppm}$ in Trockensubstanz (aus Quinche et al., 1969).

\subsection{Horizontaldistanz von der Straße}

Da der Wald schon lange für seine luftreinigende Filterwirkung bekannt ist, stellte sich die Frage, wie weit die Abgase nachweisbar seitlich in den Wald eindringen. Cannon und 
Bowles (1962) hatten in offenem Land eine etwa $50 \mathrm{~m}$ breite Randzone nachgewiesen. Unsere Untersuchungen in einem stufig geschlossenen Nadelwald zwischen Bremgarten und Wohlen $A G$ ergaben, daB im Wald der Bleigehalt mit zunehmender Distanz viel stärker abfällt als im Freiland (vgl. Abb. 3). Im Wald ist eine konstante Tendenz der Bleiabnahme mit zunehmender Distanz festzustellen (Abb. 3, Fichte), während dies für das Freiland nicht unbedingt gilt. Quinche et al. (1969) machten nämlich die überraschende Feststellung, daß im Hochsommer (Abb. 3, Emd) in der Umgebung stark befahrener Autostraßen außer am Straßenrand ein zweites Bleigehaltsmaximum in $30-100 \mathrm{~m}$ (je

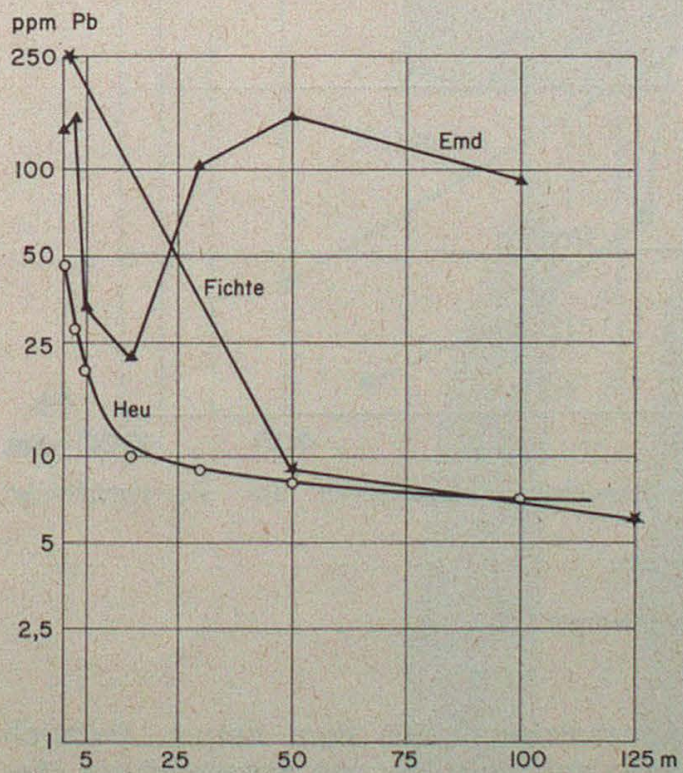

Abb. 3 Bleigehaite von Gras und Fichtennadeln in verschiedener Entfernung von der Straße.

"Fichte»: ein- und zweijährige, ungewaschene Fichtennadeln, $1 \mathrm{~m}$ über Boden, im Wohlener Wald. Straße Zürich-Bern, rund 770 Fahrzeuge/h.

"Heun: Gras des 1. Schnittes (10.6.1968) bei Nyon. Autobahn Genf-Lausanne, rund 17000 Fahrzeuge/Tag. "Emd": Gras des 2. Schnittes (27.8.1968) am selben Ort. (Aus Quinche et al., 1969.) nach Verhältnissen) auftreten kann, vermutlich wegen veränderter Turbulenzverhältnisse (zufolge hoher Fahrgeschwindigkeit und erhöhter Temperaturen). Diese Erscheinung dürfte wesentlich zu der von Keller und Preis (1967) vermuteten und von Rühling und Tyler (1968) nachgewiesenen, großräumigen Vegetationsverbleiung beitragen.

\subsection{Vertikaldistanz vom Boden}

Da die Auspuffgase meist nur wenige Dezimeter über Boden ausgestoßen werden, stellte sich die Frage, ob die Luft auch in der Kronenhöhe mittelalter und alter Bäume trotz Verwirbelung und Verdünnung noch erheblich verschmutzt sei. Im Wald ist ja selbst bei geschlossenen Bestandesrändern eine seitliche Ausbreitung der Gase möglich, im Gegensatz zu den Verhältnissen in den Städten mit geschlossenen Häuserfluchten, wo sich oft Bewohner der dritten und vierten Stockwerke noch über Abgasgestank beklagen. Die Frage hat auch eine gewisse Aktualität wegen des allfälligen Bleigehaltes von Früchten hochstämmiger Apfel- und Birnbäume in Straßennähe.

In Abb. 4 ist die Abnahme des Bleigehaltes von Fichtennadeln mit der Kronenhöhe aufgezeichnet. Sie zeigt, daß der Bleigehalt vor allem in den untersten $12 \mathrm{~m}$ mit zunehmender Höhe sehr stark abfällt, und die Verhältnisse erinnern stark an die seitliche Bleiabnahme im Freiland (Abb.3, Heu). Demgegenüber fehlt eine derartige Abnahme des Bleigehaltes in der Vertikalen bei Bäumen, welche in sauberer Luft gewachsen sind (Gmünden).

\subsection{Bleigehalt des Bodens}

Der Nachweis hoher Bleigehalte in der Vegetation warf natürlich sofort die Frage auf, ob das Blei wirklich aus der Luft stamme und nicht von den Wurzeln aus dem Boden aufgenommen worden sei. Blei als Schwermetall ist jedoch in der Pflanze nur schwer beweg- 
m Asthöhe ab Boden

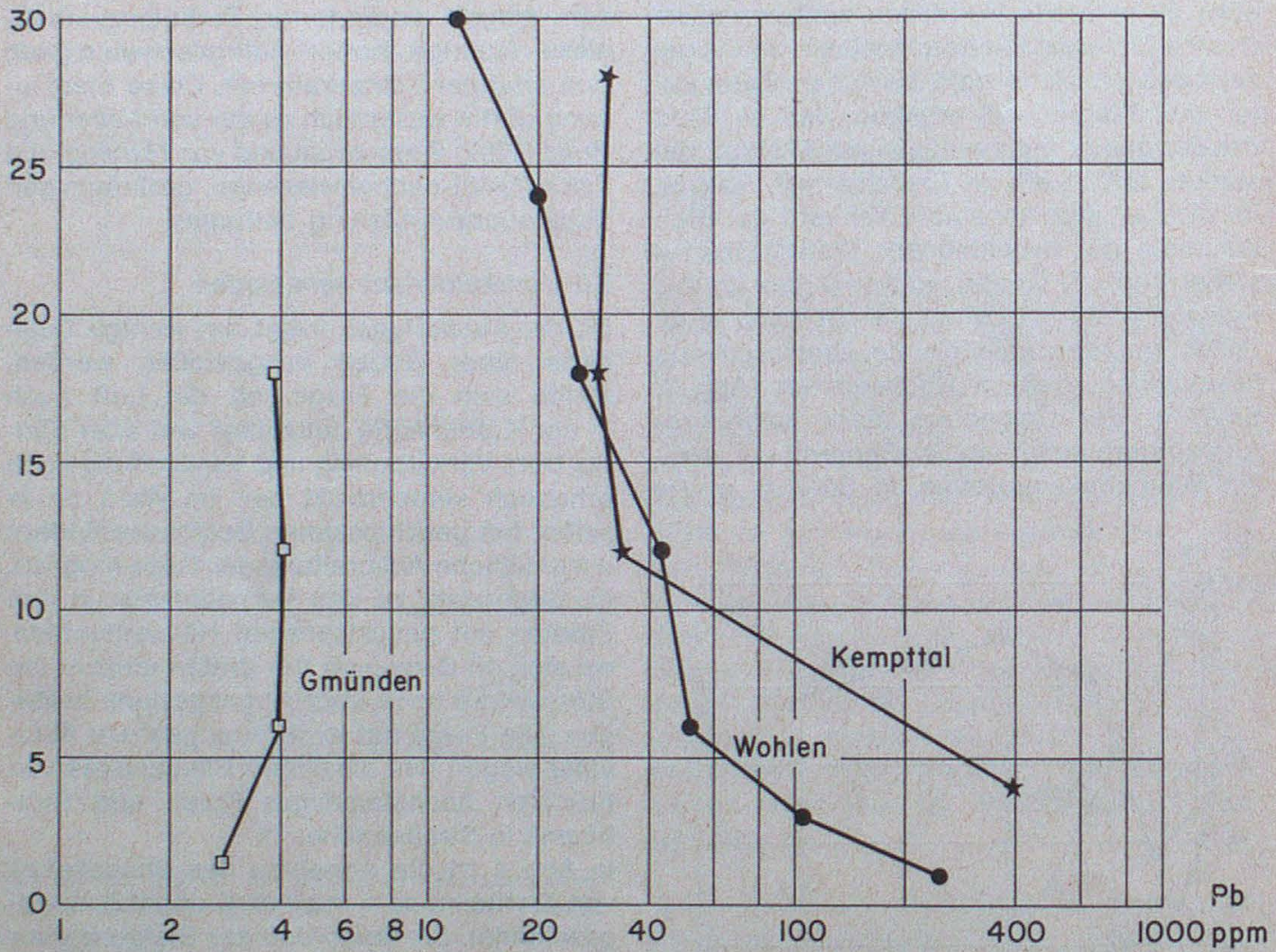

Abb. 4 Der Einfluß der Asthöhe ( $m$ über Boden) auf den Bleigehalt ungewaschener, ein- und zweijähriger Fichtennadeln.

"Kemptthal»: Straße Zürich-Winterthur, rund 965 Fahrzeuge/h.

"Wohlen": Straße Zürich-Bern, rund 770 Fahrzeuge/h.

"Gmünden": $400 \mathrm{~m}$ von autofahrbarer Straße entfernt, wenig Verkehr (AR).

lich, wie in neuerer Zeit namentlich Leh (1966) und Suchodoller (1967) mit radioaktiven Bleiverbindungen gezeigt haben.

Die mit den Auspuffgasen ausgestoßenen Bleiverbindungen besitzen nur eine geringe Löslichkeit und werden von Pflanzenwurzein nur schwer aufgenommen. Rühling und Tyler (1968) fügten in Topfversuchen dem Boden künstlich Bleioxid zu, bis dessen Blei- konzentration $2000 \mathrm{ppm}$ betrug. Dadurch stieg der Bleigehalt von Schwingelgras $(\mathrm{Fe}$ stuca ovina) nur von 5 auf 19,5 ppm. Dieselben Autoren fanden am Rande einer stark befahrenen Straße (18500 Fahrzeuge/Tag) einen totalen Bleigehalt von 300 ppm; davon waren glücklicherweise nur etwa $25 \mathrm{ppm}$ essigsäurelösliches (also etwa von Pflanzen aufnehmbares) Blei. 


\section{Die Abwaschbarkeit des Bleis}

Vom physiologischen Standpunkt aus stellt sich natürlich auch die Frage, ob das Blei nur oberflächlich angelagert wird oder ob namhafte Bleimengen in das Innere der Gewebe aufgenommen werden und dort toxisch wirken. Kloke und Riebartsch (1964) fanden, daß sich $5-30 \%$ des Bleis vom Gras abwaschen ließen, wobei mit zunehmender Entfernung von der Emissionsquelle der Pro- zentsatz des abwaschbaren Bleis abnahm. Auch nach Leh (1966) soll es sich vor allem um eine Oberflächenkontamination handeln. Suchodoller (1967) vermochte durch eine 6 stündige Waschung etwa die Hälfte des Bleis zu entfernen.

Die von uns untersuchten Fichtennadeln waren bis zu zwei Jahre den Abgasen ausgesetzt, so daß sich am Straßenrand ein krustiger Belag bildete, der auch durch eine inten-
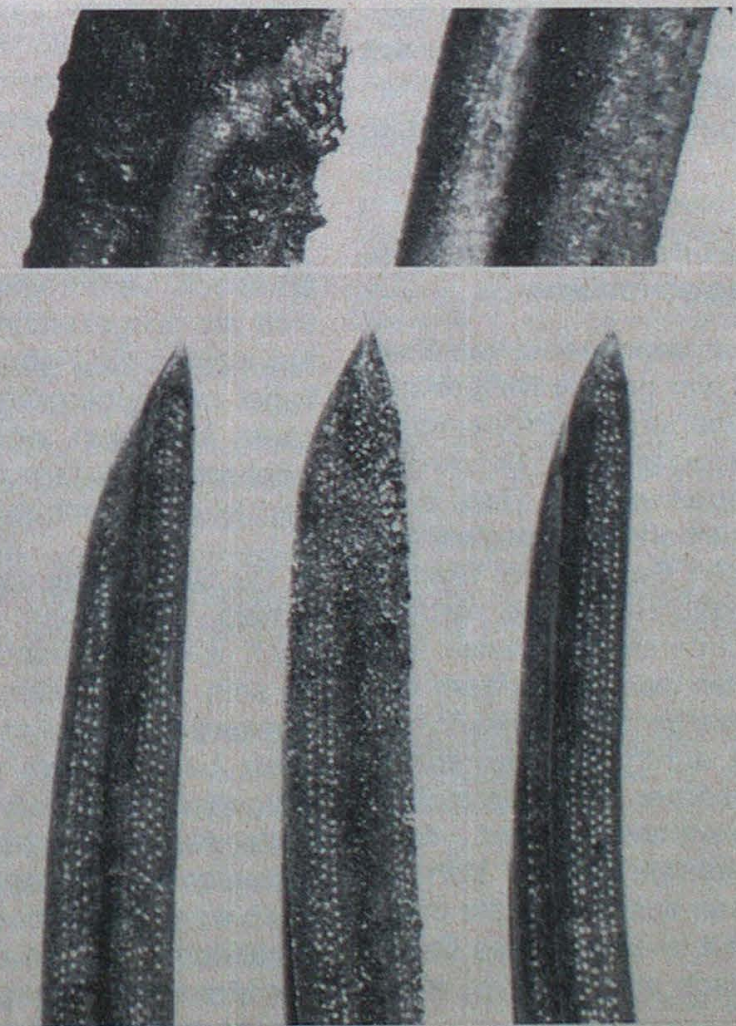

Abb. 5 Abwaschbarkeit der oberflächlich angelagerten bleihaltigen Verunreinigungen. Oben links: Nadelabschnitt mit Schmutzkruste (Nadel vom Straßenrand).

Oben rechts: schwach verschmutzter Nadelabschnitt (Nadel vom Waldesinnern).

Unten: Drei Nadeln aus einer Probe vom Straßenrand. Die mittlere Nadel wurde nicht gereinigt, die linke Nadel wurde 5 Minuten in einer einprozentigen Detergenzienlösung gewaschen; die rechte Nadel wurde je 5 Minuten in verdünnter Salpetersäure und Detergenzienlösung gewaschen. (Aus Keller und Preis, 1967.) 


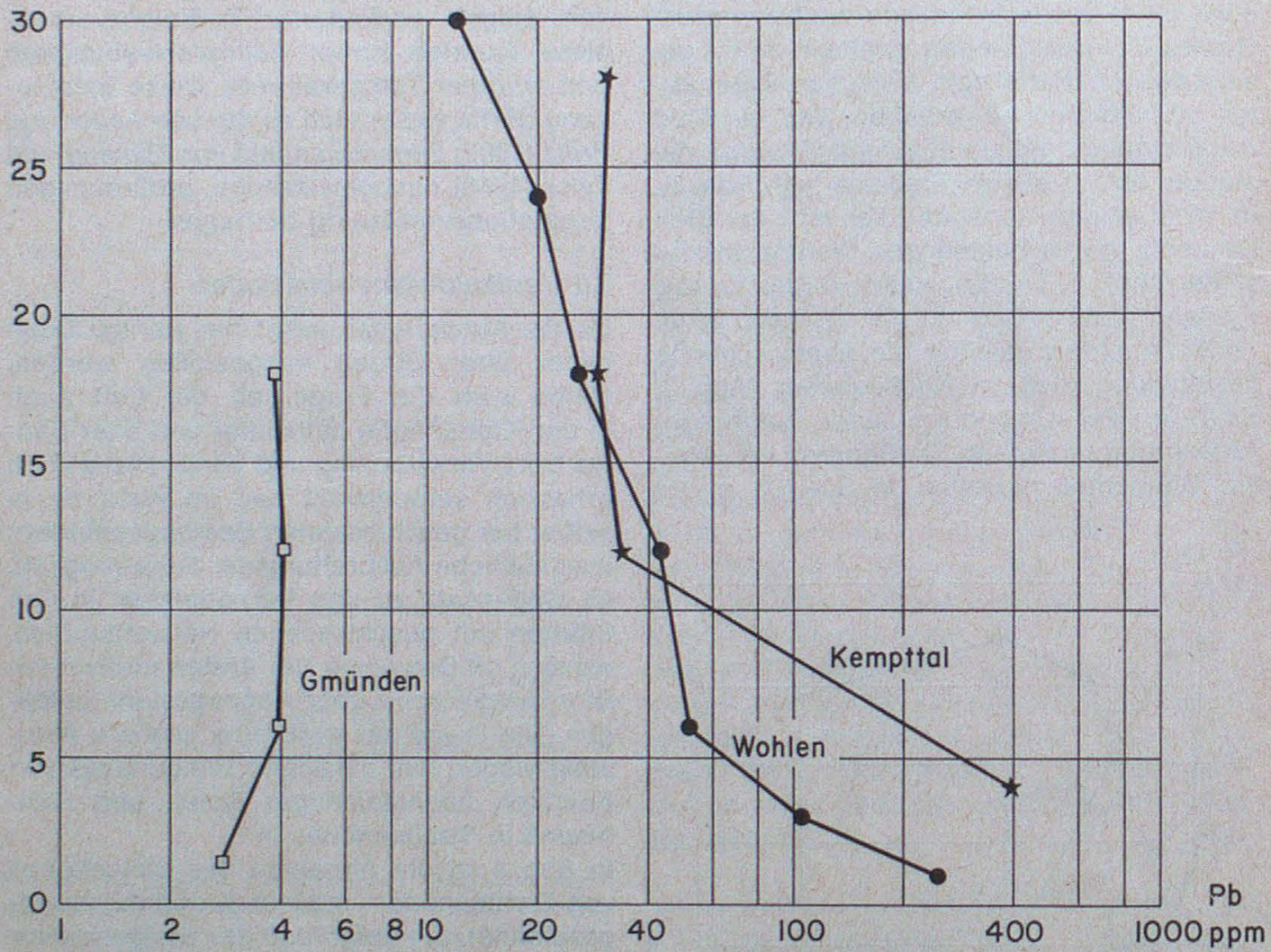

Abb. 4 Der Einfluß der Asthöhe ( $m$ über Boden) auf den Bleigehalt ungewaschener, ein- und zweijähriger Fichtennadeln.

"Kemptthal»: Straße Zürich-Winterthur, rund 965 Fahrzeuge/h.

Wohlenw: Straße Zürich-Bern, rund 770 Fahrzeuge/h.

"Gmünden": $400 \mathrm{~m}$ von autofahrbarer Straße entfernt, wenig Verkehr (AR).

lich, wie in neuerer Zeit namentlich Leh (1966) und Suchodoller (1967) mit radioaktiven Bleiverbindungen gezeigt haben.

Die mit den Auspuffgasen ausgestoßenen Bleiverbindungen besitzen nur eine geringe Löslichkeit und werden von Pflanzenwurzeln nur schwer aufgenommen. Rühling und Tyler (1968) fügten in Topfversuchen dem Boden künstlich Bleioxid zu, bis dessen Blei- konzentration $2000 \mathrm{ppm}$ betrug. Dadurch stieg der Bleigehalt von Schwingelgras ( $\mathrm{Fe}$ stuca ovina) nur von 5 auf $19,5 \mathrm{ppm}$. Dieselben Autoren fanden am Rande einer stark befahrenen Straße (18500 Fahrzeuge/Tag) einen totalen Bleigehalt von 300 ppm; davon waren glücklicherweise nur etwa $25 \mathrm{ppm}$ essigsäurelösliches (also etwa von Pflanzen aufnehmbares) Blei. 


\section{Die Abwaschbarkeit des Bleis}

Vom physiologischen Standpunkt aus stellt sich natürlich auch die Frage, ob das Blei nur oberflächlich angelagert wird oder ob namhafte Bleimengen in das Innere der Gewebe aufgenommen werden und dort toxisch wirken. Kloke und Riebartsch (1964) fanden, daß sich $5-30 \%$ des Bleis vom Gras abwaschen ließen, wobei mit zunehmender Entfernung von der Emissionsquelle der Pro- zentsatz des abwaschbaren Bleis abnahm. Auch nach Leh (1966) soll es sich vor allem um eine Oberflächenkontamination handeln. Suchodoller (1967) vermochte durch eine 6stündige Waschung etwa die Hälfte des Bleis zu entfernen.

Die von uns untersuchten Fichtennadeln waren bis zu zwei Jahre den Abgasen ausgesetzt, so daß sich am Straßenrand ein krustiger Belag bildete, der auch durch eine inten-
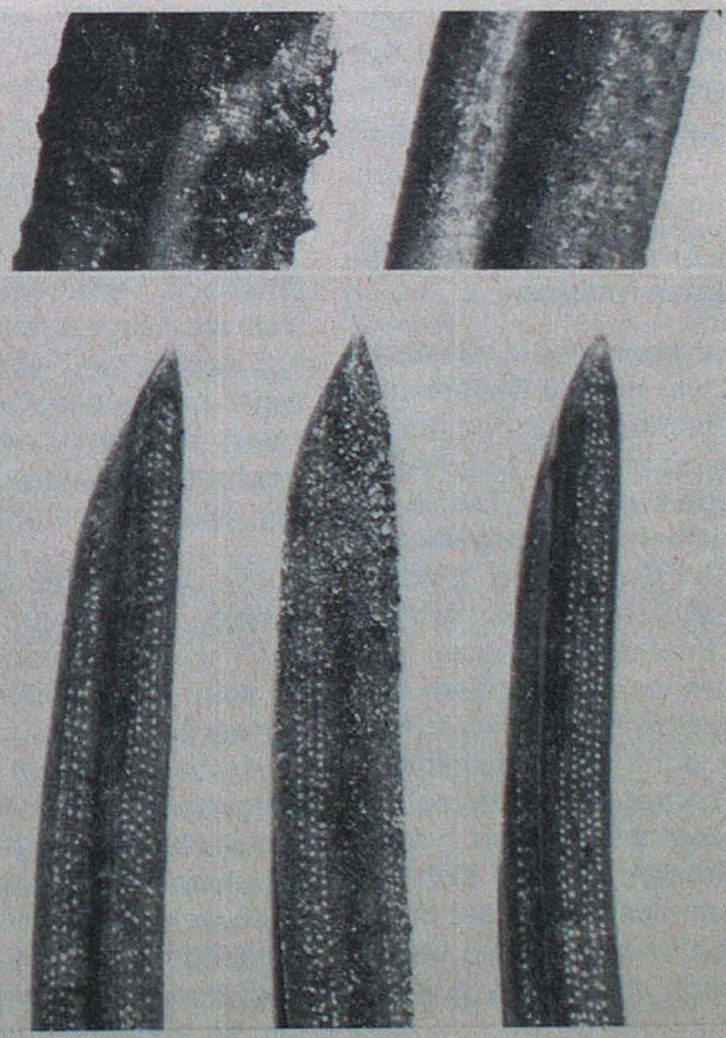

Abb. 5 Abwaschbarkeit der oberflächlich angelagerten bleihaltigen Verunreinigungen.

Oben links: Nadelabschnitt mit Schmutzkruste (Nadel vom Straßenrand).

Oben rechts: schwach verschmutzter Nadelabschnitt (Nadel vom Waldesinnern).

Unten: Drei Nadeln aus einer Probe vom Straßenrand. Die mittlere Nadel wurde nicht gereinigt, die linke Nadel wurde 5 Minuten in einer einprozentigen Detergenzienlösung gewaschen; die rechte Nadel wurde je 5 Minuten in verdünnter Salpetersäure und Detergenzienlösung gewaschen. (Aus Keller und Preis, 1967.) 